\title{
Time Series Models for Forecasting the Impact of Climate Change on Wheat Production in Varanasi District, India
}

\author{
Manvendra Singh ${ }^{1 *}$, G.C. Mishra ${ }^{1}$ and R.K. Mall ${ }^{2}$ \\ ${ }^{1}$ Department of Farm Engineering, Institute of Agricultural Sciences, BHU, \\ Varanasi-221005, India \\ ${ }^{2}$ DST-Mahamana Centre of Excellence for Climate Change Research, Institute of \\ Environment and Sustainable Development, BHU, Varanasi-221005, India \\ *Corresponding author
}

\section{A B S T R A C T}

\section{Key words \\ Climate change, \\ Autoregressive Integrated \\ Moving Average with \\ exogenous variable \\ (ARIMAX), Autoregressive \\ Integrated Moving Average \\ (ARIMA) model, Multiple \\ Linear Regression (MLR), \\ Artificial Neural Network \\ (ANN), Wheat, Root mean \\ squared error (RMSE), \\ Weather Indices \\ Article Info \\ Accepted: \\ 22 October 2018 \\ Available Online: \\ 10 November 2018}

Weather is the major threats for wheat production in South East Asia region and highly influenced by the environmental conditions, sowing date, nature of genotypes and growth stages of wheat. Climate change is a serious concern for the food security and lively hood of small farmers as reported from all over world. A period of 30 years is decided as a period of climate change study by World Meteorological Organisation (WMO).So the period from 1985-2016 is taken for the study. Weather forecasting is very important for decision making processes in management practices applying for controlling the damage caused by climate change. The objective of present study was to develop Multiple Linear Regression (MLR), Autoregressive Integrated Moving Average (ARIMA) model, Autoregressive integrated moving average with exogenous variable (ARIMAX) model, Artificial Neural Network (ANN) models for forecasting climate change impact for Varanasi region of India. For development of models, weather indices were computed from weekly data related to maximum temperature, minimum temperature, Rainfall and Solar radiation.

\section{Introduction}

Global food security threatened by climate change is one of the most important challenges in the 21 st century to supply sufficient food for the increasing population while sustaining the already stressed environment. Agriculture is sensitive to shortterm changes in weather and to seasonal, annual and longer-term variations in climate. Crop yield is the culmination of a diversified range of factors. The variations in the meteorological parameters are more of transitory in nature and have paramount influence on the agricultural systems. Analysis of the food grains production/productivity data for the last few decades reveals a tremendous increase in yield, but it appears that negative impact of vagaries of monsoon has been large throughout the period. In this context, a number of questions need to be addressed as to determine the nature of variability of 
important weather events, particularly the rainfall received in a season/year as well its distribution within the season. These observations need to be coupled to management practices, which are tailored to the climate variability of the region, such as optimal time of sowing, level of pesticides and fertilizer application. Agriculture now-a-days has become highly input and cost intensive area without judicious use of fertilizers and plant protection measures, agriculture no longer remains as profitable as before because of uncertainties of weather, production, policies, prices etc. that often lead to losses to the farmers. Under the changed scenario today, forecasting of various aspects relating to agriculture are becoming essential.

Wheat (Triticum aestivum) is one of the most extensively cultivated cereals in the world has wide adaptability to local environments. It is a winter season crop and can be grown in areas which are very hot and humid and on soils like sandy loam is considered more efficient in utilization of soil moisture and has a higher level of winter tolerance than other rabi season crops. Crop yield forecast provided useful information to farmers, marketers, government agencies and other agencies and useful in formulation of policies regarding stock, distribution and supply of agricultural produce to different areas in the country. However, statistical techniques employed should be able to provide objective crop forecasts with reasonable precisions well in advance before harvests for taking timely decisions. Various approaches have been used for forecasting such agricultural systems. The forecasting of crop yield may be done by using three major objective methods (i) biometrical characteristics (ii) agricultural inputs and (iii) weather variables. The importance of crop forecasting is more relevant in state like Uttar Pradesh which have humid - subtropical with dry winters like climate. Crop modelling can play a significant part in systems approaches by providing a powerful capability for scenario analyses. However, such forecast studies based on statistical models need to be done on continuing basis and for different agro-climatic zones, due to visible effects of changing environmental conditions and weather shifts at different locations and area. Therefore weather based forecasting models are highly reliable and cost-effective due to abrupt changes in weather in recent times. The multi feature statistical models are widely used to forecast agricultural production, price, damage caused by insect pests etc. (Zhang et al., 2003; Ho et al., 2002; Mishra and Singh 2013; Kumari et al., 2013; Paul et al., 2013; Kumari et al., 2014; Shukla et al., 2015). The objective of present study was to develop Multiple Linear Regression (MLR), Autoregressive Integrated Moving Average (ARIMA) model, Autoregressive integrated moving average with exogenous variable (ARIMAX) model, Artificial Neural Network (ANN) models for forecasting climate change impact for Varanasi region of India. A period of 30 years of weather data is decided as period for the study of impact of climate change (WMO). Nature of genotype and seeding date also influences the yield of crop. (Geleta et al., 2002), for this consideration two genotypes of wheat crop namely Sonalika and HUW 243 is taken for study in the irrigated timely sown condition.

\section{Materials and Methods}

\section{Data set}

Time series data of weather indices during 1985 to 2016, related to genotypes of wheat in Varanasi region of India was collected from annual reports of Institute of Wheat and Barley Research Karnal (Indian Council of Agricultural Research) India. Varanasi comes under North eastern plains zone (NEPZ) of India which stands second in total wheat production in India (nearly $25 \%$ ). 
The wheat production of NEPZ is affected by climate change effect, because the weather of these areas has been characterized by high temperatures and low rainfall at the late 'growth stage' of wheat crop. Since weather conditions such as high temperature and low rainfall are very favourable for progression of diseases which causes in yield loss, hence weekly weather data related to maximum temperature, minimum temperature, solar radiation and rainfall, during 1981 to 2016 were collected from India Meteorological Department, New Delhi (India).

\section{Statistical models}

\section{Multiple Linear Regressions (MLR) model}

Multiple linear regression attempts to model the relationship between two or more explanatory variables and a response variable by fitting a linear equation to observed data. In this study MLR model was used to establish a linear relationship between weekly weather parameters and spot blotch severity.Weather indices were computed from weekly weather parameters, where weights being correlation coefficient between wheat yield and weather parameters with respective weeks. Equation (2.1) and (2.2) represents the mathematical form of weather indices

$$
\begin{aligned}
& Z_{i, j}=\sum_{w=1}^{m} r_{i w}^{j} X_{i w} \\
& Z_{i, i^{\prime}, j}=\sum_{w=1}^{m} r_{i i^{\prime} w}^{j} X_{i w} X_{i^{\prime} w}
\end{aligned}
$$

Where

$J=0$, or 1 (where, ' 0 ' represents un-weighted indices and ' 1 ' represents weighted indices), w represents week number $(1,2 \ldots m)$

$r_{i w}$ is the Correlation coefficient between disease severity and $i^{\text {th }}$ weather variable in $w^{\text {th }}$ week, $r_{i i}{ }^{\prime} w$ is Correlation coefficient between disease severity/wheat yield and the product of $i$ and $i^{\text {th }}$ weather variable of $w^{\text {th }}$ week

$X_{i w}$ is the $i$ weather variables in $w^{\text {th }}$ week respectively.

\section{MLR Model}

The mathematical equation of multiple linear regression (MLR) model, as follows:

$$
Y=A_{0}+\sum_{i=1}^{p} \sum_{j=0}^{1} a_{i, j} Z_{i, j}+\sum_{i \neq i^{\prime}=1}^{p} \sum_{j=0}^{1} a_{i . i^{\prime} \cdot j} Z_{i, i^{\prime}, j}+e
$$

Where,

$Z_{i, j}$ and $Z_{i, i^{\prime}, j}$ weather indices obtained by equation (2.1) and (22), $i, i^{\prime}: 1,2, \ldots \mathrm{p}$

$p$ : Number of weather variables under study

$Y$ : Dependent Variable

$A_{0}$ : Intercept

$e$ : Error term normally distributed with mean zero and constant variance.

\section{Autoregressive integrated moving average with exogenous variable (ARIMAX) Model}

Autoregressive integrated moving average with exogenous variable (ARIMAX) is the generalization of ARIMA (Autoregressive integrated moving average) models. Simply an ARIMAX model is like a multiple regression model with one or more autoregressive (AR) term and one or more moving average terms. This model is capable of incorporating an external input variable. Identifying a suitable ARIMA model for endogenous variable is the first step for building an ARIMAX model. Testing of stationarity of exogenous variables is next step. Then transformed exogenous 
variable is added to the ARIMA model in the next step (Bierens 1987).

An ARIMA model is usually stated as ARIMA (p, d, q), where ' $p$ ' stands for the order of autoregressive process, ' $d$ ' is the order of the data and $\mathrm{q}$ is the order of the moving average process (Box and Jenkins ;1970). The general form of the ARIMA (p, d, q) can be written as

$\Delta^{d} y_{t}=\delta+\theta_{1} \Delta^{d} y_{t-1}+\theta_{2} d_{t-2}^{d} y_{t}+\cdots \ldots \ldots \ldots+\theta_{p} y_{t-p}+e_{t-1} a_{1} e_{t-1}-a_{2} e_{t-2} a_{q} e_{t-2}$

Where, $\Delta^{d}$ denotes differencing of order d, i.e. $\Delta y_{t}=y_{t}-y_{t-1} \Delta^{2} y_{t}=\Delta y_{t}-\Delta y_{t-1}$

In ARIMAX model we simply adds a new exogenous variable, in right hand side

$\Delta^{d} y_{t}=\delta+\beta x_{t}+\theta_{1} \Delta^{d} y_{t-1}+\theta_{2} d_{t-2}^{d} y_{t}+\cdots+\theta_{p} y_{t-p}+e_{t-1} a_{1} e_{t-1}-a_{2} e_{t-2} a_{q} e_{t-2}$

Where

$x_{t}$ is exogenous variable and $\beta$ is its coefficient.

Autoregressive Integrated Moving Average (ARIMA)

ARIMA is the forecasting models for nonstationary time series analysis. In contrast to the regression models, the ARIMA model allows time series to be explained by its past or lagged values and stochastic error terms. The models developed by this approach are usually called ARIMA models because they use a combination of autoregressive (AR), integration (I) - referring to the reverse process of differencing to produce the forecast and moving average (MA) operations. An ARIMA model is usually stated as ARIMA (p, $d$, q), where ' $p$ ' stands for the order of autoregressive process, ' $\mathrm{d}$ ' is the order of the data and $q$ is the order of the moving average process. The general form of the ARIMA ( $p$, d, q) can be written as

$\Delta^{d} y_{t}=\delta+\theta_{1} \Delta_{t-1}^{d} y_{t}+\theta_{2} \Delta^{d} y_{t-2}+\cdots \ldots \ldots \ldots+\theta_{p} y_{t-p}+e_{t-1} a_{1} e_{t-1}-a_{2} e_{t-2} a_{q} e_{t-2}$

Where, $\Delta^{d}$ denotes differencing of order d, i.e. $\Delta y_{t}=y_{t}-y_{t-1} \Delta^{2} y_{t}=\Delta y_{t}-\Delta y_{t-1}$ and so forth, ${ }^{y_{t-1}}$------- $y_{t-p}$ are past observations (lags), $\delta, \theta_{1}, \theta_{2} \ldots \theta_{p}$ are parameters (constant and coefficient) to be estimated similar to regression coefficient of the Auto Regressive process (AR) of order " $p$ " denoted by AR (p) and is written as

$Y=\delta+\theta_{1} y_{t-1}+\theta_{2} \ldots \ldots+\theta_{p} y_{t-p}+e_{t}$

Where $e_{t_{\text {is }}}$ the forecast error, ${ }^{\alpha_{1}}, \ldots \ldots \ldots \ldots{ }^{\alpha_{q}}$ are moving average (MA) coefficients that need to be estimated. MA model of order q i.e. MA (q) can be written as

$Y_{t}=e_{t}-a_{1} \alpha_{t-1}-a_{2} e_{t-2} \pm \ldots \ldots \ldots-a_{q} e_{t-q}(2.8)$

The major problem in ARIMA is to choose the most appropriate values for the $\mathrm{p}, \mathrm{d}$, and $\mathrm{q}$ (Figure 2). This problem can be partially resolved by looking at the Auto correlation function (ACF) and partial Auto Correlation Functions (PACF) for the series. Difference term (d) i.e. the number of time series to be differenced to yield a stationary series was determined on the basis of the value of ACF approaching to zero. After determining " $\mathrm{d}$ ", the stationary series $\Delta_{y t}^{d}$ its autocorrelation function and partial autocorrelation were examined to determine values of $\mathrm{p}$ and $\mathrm{q}$.

\section{Artificial Neural Network (ANN) Model}

ANNs are computational structures modeled on the gross structure of Brain. It is one of the model that is able to approximate various 
nonlinearities in the data. These Nonlinear autoregressive models are extensively used statistical forecasting model for time series (Hwang et al., 1994, Kapetanios 2006). The forecasting model takes the structure as follows:

$y(t) \approx f(y(t-1), y(t-2), \ldots \ldots . . . y(t-d))$

Where $y(t)$ is the forecasted output and $f_{\text {is an }}$ unknown function of the previous known outputs. Traditionally, function $f$ is determined by statistical optimization processes, such as the minimum mean squared method (Figure 1).

The feed forward neural network has been used to establish, artificial neural network models, in which the traditional function is replaced by a number of nodes that work together to implicitly approximate the same functionality [Liang 2005; Pawlus et al., 2012] as

$$
y(t) \approx \sum_{i} b_{i} \varphi\left(\sum_{j=1}^{d} a_{j i} y(t-j)\right)
$$

Where ${ }^{\varphi}$ is the transfer functions; ${ }^{a_{j i}}$ denotes the input to hidden layer weights at the hidden neuron $j$; and ${ }^{b_{i}}$ is the hidden-to-output layer weight. This is a time-delay and recurrent neural network model. The input is the known time series which is fed to the hidden layer as input according to the number of time delay.

Training set, Validation set and Test set are three main aspects of ANN. Training set is the one that has to use for the training of the algorithm. Validation set is used to find out how accurate the Algorithm is, to calculate the efficiency of the algorithm in terms of Root mean squared error (RMSE).

\section{Algorithm for ANN}

In the present study, Levenberg Marquardt (LM) algorithm was used in the development process of ANN.

LM algorithm blends the steepest descent method and Gauss -Newton algorithm.

If Sum of square due to error (SSE) for the training process is

$E(x, w)=\frac{1}{2} \sum_{p=1}^{P} \sum_{m=1}^{M} e^{2} p . m$

Where, $P$ is the number of patterns, $M$ is the number of outputs, $e_{p, m}$ is the training error at output $m$ when applying pattern $p$ and it is $e_{p, m}=d_{p, m}-o_{p, m} d$ is the desired output vector and $o$ is the actual output vector.

In the Steepest Descent Algorithm, update rule of weights is: $w_{k+1}=w_{k}-\alpha \mathrm{g}_{k}$, where $k$ is the index of iterations, $x$ is the input vector and $w$ is the weight vector, $\alpha$ is the learning constant (step size) and $g$ is gradient. Whereas, in the Newton's Method, update rule for Newton's method is: $w_{k+1}=w_{k}-H_{k}^{-1} g_{k}$, where $\mathrm{H}$ (square matrix) is the Hessian matrix given as:

$H=\left[\begin{array}{lccc}\frac{\partial^{2} E}{\partial w_{1}^{2}} & \frac{\partial^{2} E}{\partial w_{1} \partial w_{2}} & \cdots & \frac{\partial^{2} E}{\partial w_{1} \partial w_{\mathrm{N}}} \\ \frac{\partial^{2} E}{\partial w_{2} \partial w_{1}} & \frac{\partial^{2} E}{\partial w_{2}^{2}} & \cdots & \frac{\partial^{2} E}{\partial w_{2} \partial w_{\mathrm{N}}} \\ \cdots & \cdots & \cdots & \ldots \\ \frac{\partial^{2} E}{\partial w_{N} \partial w_{1}} & \frac{\partial^{2} E}{\partial w_{N} \partial w_{2}} & \cdots & \frac{\partial^{2} E}{\partial w_{2} \partial w_{N}^{2}}\end{array}\right]$

In Gauss-Newton Algorithm, update rule of weights is

$$
w_{k+1}=w_{k}-\left(J_{k}^{T} J_{k}\right)^{-1} J_{k} e_{k}
$$


Where, $J$ is Jacobian matrix, defined as;

$$
J=\left[\begin{array}{llll}
\frac{\partial^{2} e_{1.1}}{\partial w_{1}} & \frac{\partial^{2} e_{1.1}}{\partial w_{2}} & \ldots & \frac{\partial^{2} e_{1.1}}{\partial w_{\mathrm{N}}} \\
\frac{\partial^{2} e_{1.2}}{\partial w_{1}} & \frac{\partial^{2} e_{1.2}}{\partial w_{2}} & \ldots & \frac{\partial^{2} e_{1.2}}{\partial w_{\mathrm{N}}} \\
\cdots & \ldots & \ldots & \ldots \\
\frac{\partial^{2} e_{1 . M}}{\partial w_{1}} & \frac{\partial^{2} e_{1 . M}}{\partial w_{2}} & \ldots & \frac{\partial^{2} e_{1 . M}}{\partial w_{\mathrm{N}}} \\
\cdots & \cdots & \ldots & \ldots \\
\frac{\partial^{2} e_{P .1}}{\partial w_{1}} & \frac{\partial^{2} e_{P .1}}{\partial w_{2}} & \ldots & \frac{\partial^{2} e_{P .1}}{\partial w_{\mathrm{N}}} \\
\frac{\partial^{2} e_{P .2}}{\partial w_{1}} & \frac{\partial^{2} e_{P .2}}{\partial w_{2}} & \ldots & \frac{\partial^{2} e_{P .2}}{\partial w_{\mathrm{N}}} \\
\cdots & \cdots & \ldots & \ldots \\
\frac{\partial^{2} e_{P . M}}{\partial w_{1}} & \frac{\partial^{2} e_{P . M}}{\partial w_{2}} & \cdots & \frac{\partial^{2} e_{P . M}}{\partial w_{\mathrm{N}}}
\end{array}\right]
$$

Where error vector $\boldsymbol{e}$ has the form $\mathrm{e}^{\mathrm{T}}=\left[\mathrm{e}_{1,1} \mathrm{e}_{1,2} \ldots \mathrm{e}_{1, \mathrm{M}} \ldots \ldots . \mathrm{e}_{\mathrm{P}, 1} \mathrm{e}_{\mathrm{P}, 2 \ldots} \mathrm{e}_{\mathrm{P}, \mathrm{M}}\right](2.15)$

In order to make sure that the approximated Hessian matrix $\boldsymbol{J}^{T} \boldsymbol{J}$ is invertible, LevenbergMarquardt algorithm introduced another approximation to Hessian matrix:

$$
H=J^{t} J+\mu I
$$

Where $\mu$ is called combination coefficient, which is always positive and $I$ is the identity matrix.

So the update rule of weights in this algorithm is:

$$
w_{k+1}=w_{k}-\left(J_{k}^{T} J_{k}+\mu I\right)^{-1} J_{k} e_{k}
$$

[Hao et al., 2011].

\section{Accuracy Measurement of the Model}

To make comparison of forecasting ability among models is Root mean square Error (RMSE) given as:
RMSE $=\sqrt{\frac{\sum\left(p_{t}-A_{\mathrm{t}}\right)^{2}}{T}}$

Where,

T: Total number of observations in the time series.

$\mathrm{P}_{\mathrm{t}}$ : Predicted Value at time $\mathrm{t}$.

At: Actual value at time t.

\section{Results and Discussion}

Time series data (1985-2016) of monthly average maximum temperature (in ${ }^{0} \mathrm{C}$ ), monthly average minimum temperature (in $\left.{ }^{0} \mathrm{C}\right)$, monthly average rainfall $(\mathrm{mm})$ and solar radiation $\left(\mathrm{MJ} / \mathrm{m}^{2}\right)$.

\section{Multiple Linear Regression (MLR)}

Multiple Regression model widely used forecasting model. In Multiple Regression Model (MLR) $\mathrm{Y}=$ Dependent variable and $\mathrm{X}_{1}$, $\mathrm{X}_{2}, \mathrm{X}_{3} \ldots$. Used as independent variable.

Different regression models are fitted during the growing period of wheat. Wheat growing weeks which are important from the yield point of view are chosen to fit the model. On the basis of this point $3^{\text {rd }}, 6^{\text {th }}, 10^{\text {th }}, 11^{\text {th }}, 13^{\text {th }}$ and $16^{\text {th }}$ weeks are chosen for fitting of regression models.

Regression equations, RMSE and R-Square values are given in the Table 1. With their respective MLR equations.

\section{Autoregressive integrated moving average with exogenous variable (ARIMAX) Model}

On the basis of data yearly production and weekly weather parameters were calculated for measuring the quantitative relationship between these variables. 
Table.1

\begin{tabular}{|l|l|c|c|}
\hline Week & MLR models for forecasting climate change effect & RMSE & R- Square value \\
\hline $3^{\text {rd }}$ & $Y=4741.42-316.86 X_{1}+130.91 X_{2}-113.22 X_{3}$ & 188.83 & 17.58 \\
\hline $6^{\text {th }}$ & $Y=-1134-603.94 X_{1}-296.31 X_{2}+249.69 .22 X_{3}+135.29 X_{4}$ & 193.28 & 9.21 \\
\hline $10^{\text {th }}$ & $Y=2923.28-82.09 X_{1}+38.44 X_{2}-26 X_{3}+29.85 X_{4}$ & 192.66 & 18.90 \\
\hline $1^{\text {th }}$ & $Y=2721.63-0.18 X_{1}-28.20 X_{2}-45.22 X_{3}-131.17 X_{4}$ & 164.85 & 33.95 \\
\hline $1^{\text {th }}$ & $Y=5123.28-819.45 X_{1}+454.32 X_{2}-472.98 X_{3}-19.87 X_{4}$ & 182.28 & 19.25 \\
\hline $1^{\text {th }}$ & $Y=2320.28-3.20 X_{1}+2.01 X_{2}-1.75 X_{3}-21.56 X_{4}$ & 190.77 & 15.22
\end{tabular}

Fig.1 Regression plot for ANN model
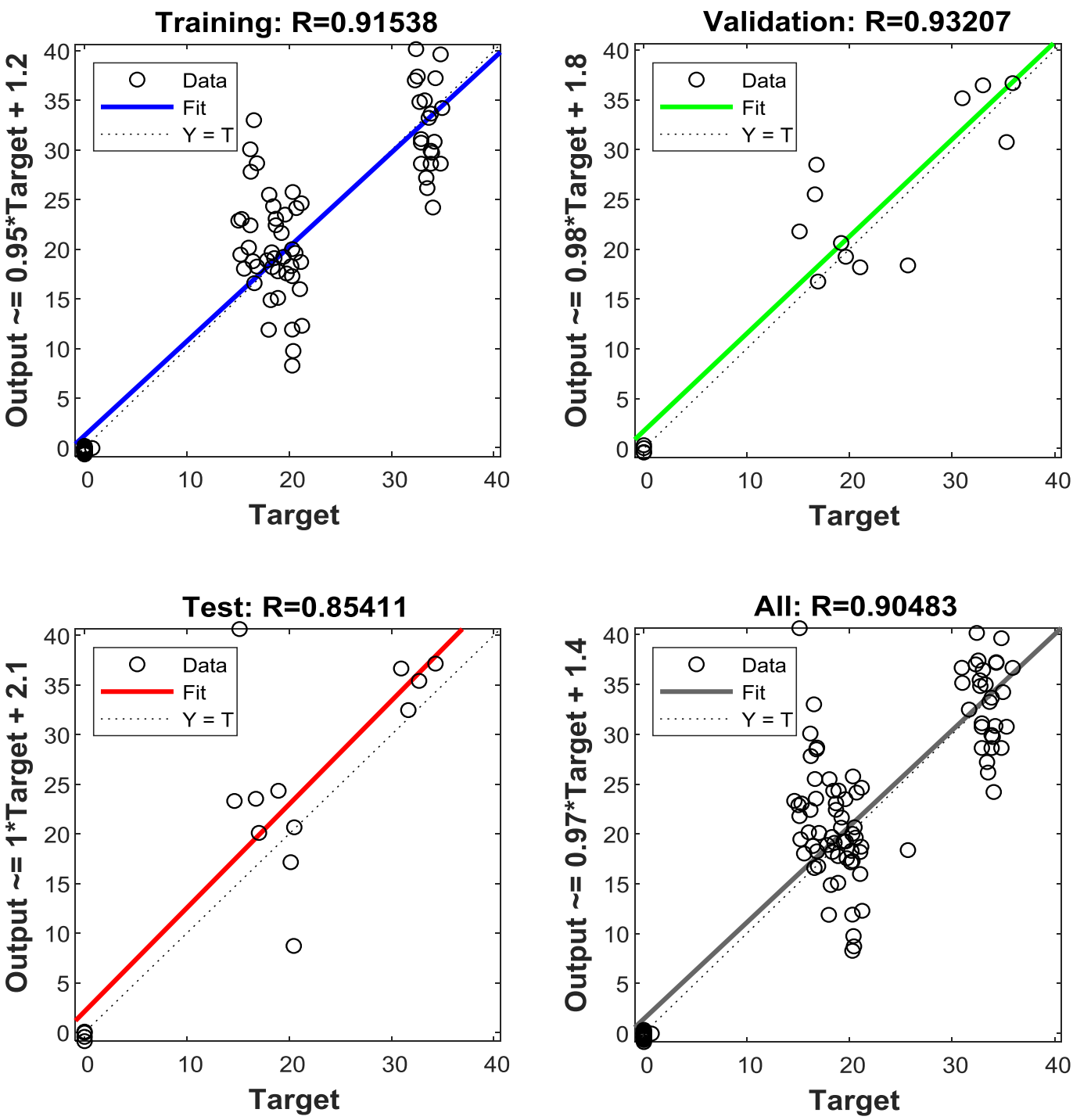
Fig.2 Flow chart of Box-Jenkins Methodology

\section{Stage-1 Identification}

Stage- 2 Estimation

Stage-3 Diagnostic Checking
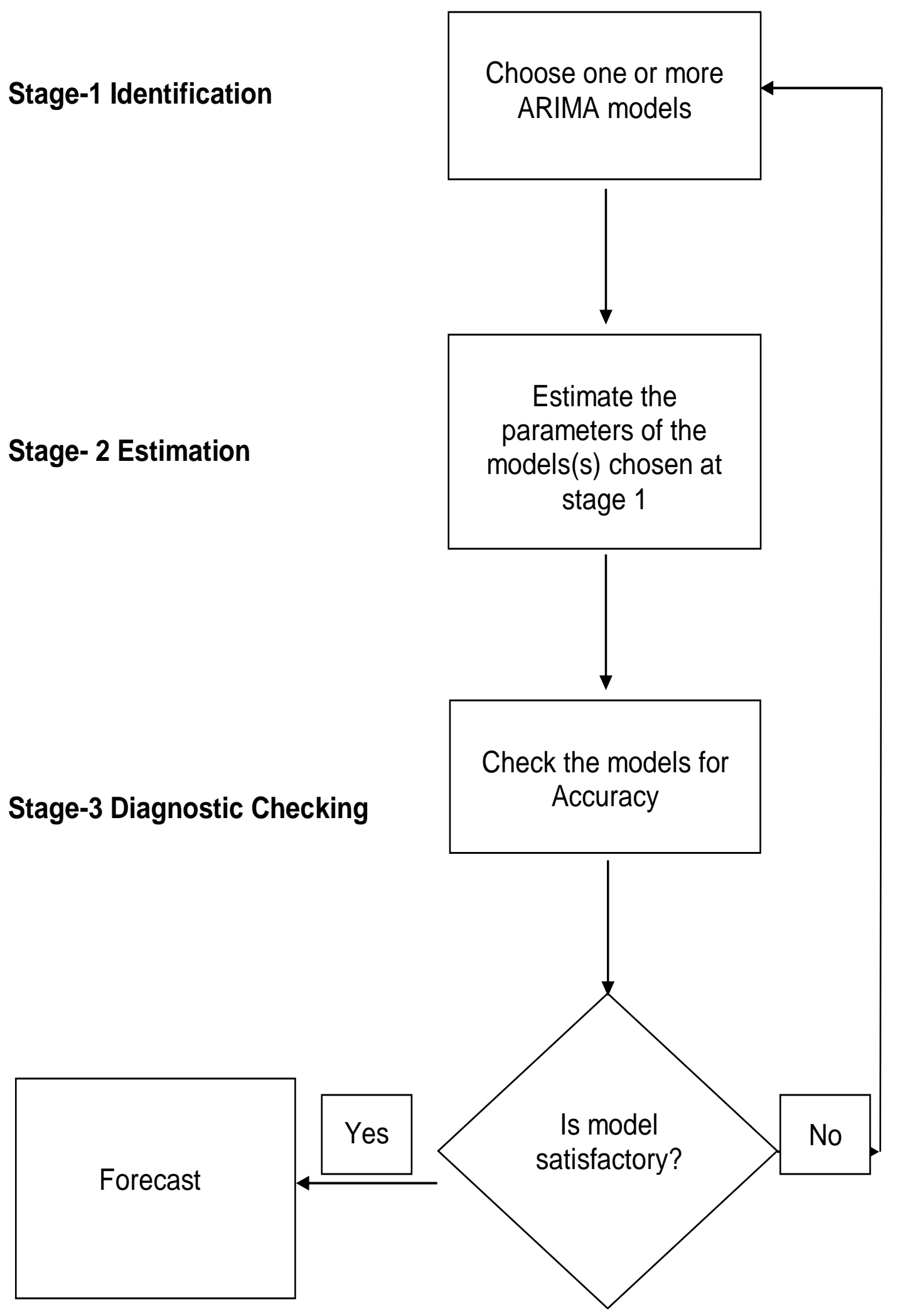

Estimate the parameters of the models(s) chosen at stage 1 
ARIMAX model $(2,0,2)$ is the best fit model among all other ARIMAX models. This has the highest $\mathrm{R}$ - Square value which is 37.4 with lowest RMSE value 367.9 which is lowest among all other models from. Among all models ARIMAX model $(2,0,2)$ is the best fitted model for forecasting of production with highest $\mathrm{R}$ - Square value of 37.4 .

\section{Autoregressive Integrated Moving Average (ARIMA)}

On the basis of data yearly production and weekly weather parameters were calculated for measuring the quantitative relationship between these variables

ARIMA model $(3,0,3)$ is the best fit model among all other ARIMAX models. This has the highest $\mathrm{R}$ - Square value which is 21.4 with lowest RMSE value 324.9 which is lowest among all other models From. Among all models ARIMAX model $(3,0,3)$ is the best fitted model for forecasting of production with highest R - Square value of 21.4.

\section{ANN (Artificial Neural Network)}

On the same data set ANN model used. After a lot of training of the ANN best fit model is choosen. The best fit model of the ANN is with the R - Square value 93.20 and RMSE value 186.4.

Since in Varanasi, timely sowing of wheat crop may be done in the first fort night of November month, hence daily weather data (maximum temperature, minimum temperature, rainfall and solar radiation) from November to March was considered for the study.

It is seen from the comparison of $\mathrm{R}$ - Square value and RMSE of the models. In the study it is clear that ANN is the best fit model for forecasting of the impact of climate change in
Varanasi district but for the some wheat growing weeks MLR model also predicts better than ANN. ARIMA and ARIMAX models are also better than MLR for overall prediction but they are having more RMSE value than the weekly MLR models but ANN models always have highest R-Square value and low RMSE than all other models so ANN is the best model to fit.

\section{Acknowledgement}

The authors are thankful to the ICAR-Indian Institute of Wheat and Barley Research Karnal (Indian Council of Agricultural Research) India and India Meteorological department for providing data to carry out the present study.

\section{References}

Agrawal, Ranjana; Jain, R.C. and Jha, M.P. (1983). Joint effects of weather variables on rice yield, Mausam, 34 (2): 189-94.

Agrawal, Ranjana; Jain, R.C. and Jha, M.P. (1986). Models for studying rice cropweather relationship, Mausam, 37(1): 67-70.

Agrawal, Ranjana; Jain, R.C. and Mehta, S.C. (2001). Yield forecast based on weather variables and agricultural inputs on agro climatic zone basis. Indian Journal of Agricultural Science, 71(7).

Agrawal, Ranjana; Jain, R.C.; Jha, M.P. and Singh, D. (1980). Forecasting of rice yield using climatic variables. Ind.J.Agri. Sci. 50(9): 680-84.

Agrawal, Ranjana; Ramakrishna, Y.S.; Kesava Rao, A.V.R.; Kumar, Amrender; Bhar, Lal Mohan; Madan Mohan and Saksena, Asha (2005). Modeling for forecasting of crop yield using weather parameter and agricultural inputs. 
Bierens H J. 1987. ARMAX model specification testing, with an application to unemployment in the Netherlands. Journal of Econometrics 35: 161-90.

Box, G.E.P. and Jenkins, G.M. (1970).Time Series Analysis: Forecasting and Control. San Francisco: Holden-Day.

Dubin, H.J. 1984. Regional and in-country activities: Andean region, Report on wheat improvement 1981.CIMMYT, Mexico D.F.:102-104

Ho, S.L., Xie, M. and Goh, T.N., 2002. A comparative study of neural network and Box-Jenkins ARIMA modeling in time series prediction, Computers and Industrial Engineering 42: 371- 375.

Hwang S. Y. and Basawa, I. V. 1994. Large sample inference based on multiple observations from nonlinear autoregressive processes. Stochastic Processes and their Applications, 49: 127-140

Kiesling, RL. (1985) the diseases of barley. In: Rasmusson DC (ed) Barley agron monogr 16. ASA and SSSA, Madison, WI, pp 269-308

Kumari P., Mishra G.C. and Srivastava C.P. 2013. Forecasting of productivity and pod damage by Helicoverpa armigera using artificial neural network model in Pigeonpea (Cajanus cajan). International Journal of Agriculture, Environment and Biotechnology.6.335340.

Kumari P., Mishra GC and Srivastava CP. 2014. Time series forecasting of losses due to pod borer, pod fly and productivity of pigeonpea (Cajanus cajan) for North West Plain Zone (NWPZ) by using artificial neural network (ANN). International Journal of Agricultural and Statistical Science 10: $15-21$

Mishra GC and Singh A 2013. A study on forecasting, prices of groundnut oil in Delhi by ARIMA methodology and Artificial Neural Networks. AGRIS online papers in Economics and Informatics 5:25-34.

Paul R.A., Prajneshu, Ghosh H. (2013). Statistical modelling for forecasting of wheat yield based on weather variables. Indian Journal of Agricultural Sciences. 83. 60-63.

Saari, E.E. 1998. Leaf blight disease and associated soil borne fungal pathogens of wheat in South and South East Asia. E. Duveiller, H. J. Dubin, J. Reeves and A. McNab (eds.), Helminthosporium blight of wheat: spot blotch and tan spot. CIMMYT, Mexico. D.F:37-51.

Shukla G., Mishra G.C., Singh S.K. 2015. Kriging approach for estimating deficient micronutrients in the soil: A case study. International Journal of Agriculture, Environment, and Biotechnology. 8: 309-314.

Zhang, G.P., 2003. Time series forecasting using a hybrid ARIMA and neural network model. Neurocomputing 50: 159-175.

\section{How to cite this article:}

Manvendra Singh, G.C. Mishra and Mall, R.K. 2018. Time Series Models for Forecasting the Impact of Climate Change on Wheat Production in Varanasi District, India. Int.J.Curr.Microbiol.App.Sci. 7(11): 2687-2696. doi: https://doi.org/10.20546/ijcmas.2018.711.307 